\title{
Desigualdades digitales y el modelo 1 a 1 como solución. El caso de One Laptop Per Child Perú (2007-2012)
}

\author{
Ana Laura Rivoir ${ }^{1}$ [1] @ \\ ${ }^{1}$ Universidad de la República, Uruguay
}

Resumen. El concepto de brecha digital proviene inicialmente de la dicotomía de acceso o no acceso a las tecnologías digitales; incorporando después los distintos tipos de usos, la apropiación y los resultados sobre la vida de las personas; y, el desarrollo de competencias digitales para el aprovechamiento de estas tecnologías como fin del desarrollo individual y colectivo. Esta evolución ha ido transformando las políticas. El artículo analiza el caso de la implementación del One Laptop Per Child Perú (20072012). Su aplicación, la concepción que lo fundamenta y cómo se adaptó la propuesta al contexto. Se sustenta en la información producida por una investigación realizada en 2015 y otros datos secundarios, documentos políticos, datos estadísticos y entrevistas a actores de estas políticas tanto a nivel central como de los centros educativos. Se concluye que, a pesar del apoyo político al más alto nivel y a la inversión realizada, factores como la debilidad institucional, la infraestructura tecnológica deficiente y la inadecuación de la tecnología al contexto son factores insalvables en términos de resultados. Se refutó la premisa del OLPC acerca del aprendizaje autónomo de los estudiantes para la apropiación de tecnología en beneficio de procesos de aprendizaje. Palabras clave: inclusión digital; educación, desigualdades sociales; política pública.

Desigualdades digitais e o modelo 1 a 1 como solução. O caso de One Laptop Per Child Peru (2007-2012).

Resumo. O conceito de exclusão digital deriva inicialmente da dicotomia de acesso ou não acesso às tecnologias digitais; incorporando posteriormente os diferentes tipos de usos, a apropriação e os efeitos na vida das pessoas; e o desenvolvimento de competências digitais para o uso dessas tecnologias a fim de alcançar o desenvolvimento individual e coletivo. Esta evolução tem transformado as políticas.

O artigo analisa o caso da implementação do One Laptop Per Child Peru (2007-2012), a sua aplicação, a concepção que o sustenta e a maneira que a sua proposta foi adaptada ao contexto. O estudo fundamenta-se nas informações geradas em uma pesquisa realizada em 2015 e outros dados secundários, documentos políticos, dados estatísticos e entrevistas com atores destas políticas tanto no âmbito central quanto nas instituições de ensino.

Conclui-se que, apesar do apoio político ao mais alto nivel e ao investimento realizado, fatores como a fragilidade institucional, a infraestrutura tecnológica deficiente e a inadequação da tecnologia ao contexto são condições insuperáveis em termos de resultados. Portanto, a premissa do OLPC sobre a aprendizagem autônoma dos estudantes para a apropriação da tecnologia em benefício dos processos de aprendizagem foi refutada. Palavras-chave: inclusão digital; educação; desigualdades sociais; política pública.

Digital inequalities and the 1 to 1 model as a solution. The case of One Laptop Per Child Peru (2007-2012).

Abstract. The digital divide concept evolves initially the dichotomy of access and not access to digital technologies, incorporating later the different types of uses, the appropriation and results on the lives of people, and the development of digital skills for the use of these digital technologies to purposes of individual and collective development. These changes transformed the policies.

The article analyses the case of the implementation of One Laptop Per Child Peru (2007-2012). Its implementation, the conception that supports it and how the proposal was adapted to the context. Based on the information produced in a research carried out in 2015 and secondary data, it analyses policy documents and statistical data, and interviews with policy actors both at the central level and in the schools.

It concludes that, despite the political support at the highest level and the investment made, factors such as institutional weakness, poor technological infrastructure and the inadequacy of technology to the context are insurmountable factors in terms of results. 
The premise of OLPC about the autonomic learning of students for the appropriation of technology for the benefit of learning processes was refuted.

Keywords: digital inclusion; education, social inequalities; Public politics

\section{Introducción}

Los cambios experimentados en las sociedades contemporáneas, alimentados por la acelerada digitalización en todos los ámbitos de la sociedad involucran a la educación, pues demanda formación y creación de capacidades. Según Castells (2002) las Tecnologías de la Información y la Comunicación (TIC) ocupan un papel central en la producción y la reproducción social, por lo que la capacidad para beneficiarse de ellas constituye una dimensión en la desigualdad social de las sociedades contemporáneas. Estas tecnologías se vinculan a beneficios personales, sociales, económicos, políticos y culturales; y, por tanto, conforman además una dimensión importante de la inclusión social (van Deursen y van Dijk, 2014).

Con el avance en el desarrollo tecnológico se evidencian las diferencias existentes, en cuanto a las posibilidades de beneficiarse del mismo, emergiendo el concepto de 'brecha digital' como una desigualdad característica de esta era digital.

Inicialmente este concepto fue concebido como la divisoria dicotómica entre quienes tenían acceso a las Tecnologías de la Información y la Comunicación (TIC) y quiénes no. Este escenario fundamentó políticas de consolidación en la infraestructura y la conectividad, bajo el supuesto que beneficiaría de forma directa y automática a los ciudadanos. En muchos casos, sustentadas por los intereses particulares de las empresas de telecomunicaciones (Mansell, 2002; Stewart et al, 2006). Este enfoque sobredimensiona la capacidad de las TIC, entendiendo que su mera adquisición logra bienestar y desarrollo social (Cortés y Dubois, 2005).

A medida que avanzan los estudios y las evaluaciones de estas políticas, se constata que las diferencias en el acceso están relacionadas con las posibilidades de los diversos grupos sociales. Se distinguen distintas dimensiones de brecha digital: la generacional; la del nivel socio-económico; la de género; la geográfica (urbana o rural); la étnica; la del nivel educativo, entre otras; y, cómo éstas replican en la reproducción de desigualdades preexistentes (Norris, 2001; Mística, 2003; Hargittai, 2004; Sassi, 2005). Un fenómeno que puede constituirse en una dinámica recursiva, que implica que la exclusión digital está dada por las desigualdades preexistentes. A la 
vez, la exclusión digital reforzaría las otras exclusiones. Este fenómeno pone en evidencia la necesidad de políticas específicas (Sassi, 2005; GascóHernández, Equiza-Lopez y Acevedo-Rui, 2007).

Por otra parte, se constata que el uso de estas tecnologías también genera diferencias y que las capacidades desiguales para aprovecharlas también crean desigualdad, lo que viene a denominarse segunda brecha digital. Ya que están relacionadas por las características sociodemográficas y también por las capacidades de apropiación de los individuos (Robinson, DiMaggio y Hargittai, 2003; van Dijk, 2005; Hargittai y Hinnant, 2008; Helsper, 2012).

La apropiación se refiere cuando las TIC se incorporan en la vida cotidiana, generando acciones y prácticas que cambian en un sentido deseado las condiciones de vida, permitiendo resolver problemas y colmar necesidades (Camacho, 2001; Warschauer, 2003; Selwyn, 2004; Rivoir, 2013). Se trata de un proceso que siempre se produce en el marco de un contexto cultural y social determinado (Bonilla y Cliche, 2001; Cortés y Dubois, 2005). Una tercera dimensión de brecha digital sería la compuesta por el acceso, el uso y la apropiación, que agregados se traducen en resultados efectivos en la vida de las personas (Rivoir y Lamschtein, 2018).

Esta evolución de la brecha digital interpela a las políticas, pues las desafía a nuevas acciones e iniciativas que contemplen la variedad de dimensiones involucradas en la conformación de esta desigualdad. Las políticas de inclusión digital deben considerar estos elementos para obtener resultados deseados.

\section{Políticas TIC en la educación para la reducción de desigualdades}

Las políticas de incorporación de TIC en la educación son pioneras. Adaptarse a estas sociedades en cambio permanente, implica no sólo el manejo de la tecnología, sino repensar las competencias básicas requeridas (Griffin et al. 2012). Según Claro et al. (2011) hay tres niveles en la que pueden intervenir las TIC en educación: a) en la gestión del centro educativo; b) el pedagógico con recursos digitales y métodos novedosos; y, c) el curricular orientado a las asignaturas y a las habilidades y competencias digitales, para facilitar la integración social y económica futura de los estudiantes. 
Esto plantea desafíos a los sistemas educativos. Selwyn (2013) sostiene que está relativamente consensuado que las tecnologías digitales deben ser incorporadas a los procesos de aprendizaje a lo largo de la vida de las personas, en los distintos niveles educativos y como formación permanente necesaria.

Los estudiantes -en su amplia mayoría- usan tecnologías digitales, pero lo hacen fundamentalmente como parte del entretenimiento y la sociabilidad, lo que no significa provechoso para su formación en un contexto educativo o para desarrollar habilidades útiles en su mejora académica. De aquí, se desprende la importancia del rol docente en las estrategias y en la metodología para usar los recursos digitales en clase (Albertos et al., 2016; García, Gros y Escofet, 2012; Duart et al., 2008). Pedró (2006) añade que en estos contextos es fundamental involucrar a los estudiantes en el sistema educativo, contando con docentes innovadores en el uso de las TIC en el aula, a efectos de propiciar el desarrollo de sujetos protagonistas de su aprendizaje, que les consoliden como productores en la red, opinando, interactuando y aportando conocimiento. Según Cabero y Llorente (2013) se debe estimular la autorregulación y responsabilidad del estudiante sobre su propio aprendizaje, favoreciendo la transformación de habilidades mentales en habilidades para su desempeño académico. Es clave la integración de las TIC en los procesos de aprendizaje para favorecer las estrategias colaborativas y la ubicuidad, promoviendo también los contextos de aprendizaje tanto formales como informales.

En América Latina las tecnologías digitales vienen introduciéndose en el sistema educativo desde los años 80 y su presencia ha ido en aumento, tanto por la incorporación de los estudiantes y docentes en su vida cotidiana, como por las políticas públicas implementadas (Rivoir, 2013 y 2017). Estas políticas han cobrado trascendencia en las estrategias digitales de los países latinoamericanos, a pesar de la prioridad otorgada en los documentos, su implementación es muy desigual. Las políticas a través de programas a nivel país o de gobiernos locales, se han centrado en una primera fase en dotar de infraestructura y soporte técnico; y, en una segunda fase en usar estas tecnologías en la gestión, la innovación en las prácticas educativas, el desarrollo de competencias docentes y en los estudiantes para su desarrollo cognitivo y su mejora en el aprendizaje (Hinostroza y Labbé, 2011).

Solo algunos países han logrado de forma continuada hacer efectivas estas políticas y acciones de TIC en educación con sostenibilidad y resultados que empiezan a ser evidentes (Jara, 2011). 
Al inicio de este milenio se comenzaron a abordar los temas pedagógicos y de formación docente, en especial los vinculados a los dispositivos portátiles. Posteriormente, surgen los primeros estudios que llaman la atención acerca de la falta de resultados contrastables en relación a los aprendizajes, lo cual frustra a los actores de las políticas (Sunkel, Trucco y Espejo, 2013).

La modalidad 1 a 1 , de la propuesta de OLPC, fue implementada en varios países como una política pública con ambiciones de universalidad, existen las experiencias de Uruguay, Argentina y Perú. De éstas, el caso del Perú es el que más críticas ha recibido, mientras que la de Uruguay es la que se toma como la más paradigmática. Aunque sus objetivos y formato son muy similares, pues se propusieron atender a los más desfavorecidos de la sociedad, y contaron con una inversión enorme y una voluntad política al mayor nivel del Estado. Ahora nos interesa saber ¿qué factores conformaron estas experiencias?, ¿cuáles fueron sus fallos y virtudes?, ¿qué beneficios aplican en la reducción de la desigualdad digital y en qué sentido?

En 2007 Perú y Uruguay fueron los primeros países que se propusieron implementar la propuesta de Un computador por Niño a nivel nacional en la educación primaria. Los resultados de ambas experiencias están siendo distintos. Mientras que en el Uruguay el Plan Ceibal sigue su curso al 2019, en el Perú la iniciativa no prosperó más allá del 2012.

Cabe señalar algunos aspectos críticos respecto a la forma de su implementación: la imposición vertical e inconsulta a los actores educativos (Cano, 2015); la falta de formación a los docentes, en particular los de las zonas rurales, cuyo conocimiento se aleja mucho de la informática (Laura, 2011); y, la poca centralidad en los aspectos educativos (Cristiá et al., 2011).

Los desafíos de esta modalidad en la incorporación de las tecnologías digitales en la educación para la reducir desigualdades, alcanza también a sus formas de evaluación, pues interpela viejos modelos y esquemas (UNESCO, 2013; Valente, 2014; Benítez y Winocur, 2016). El análisis de estas políticas es clave para comprender mejor los procesos y dinámicas de la implementación de iniciativas de inclusión digital, a partir del sistema educativo, pero con objetivos de incidencia en distintos procesos y ámbitos como la familia y el comunitario, así como los resultados en términos de su vinculación con la pobreza y los sectores más excluidos de las sociedades. 


\section{Metodología}

A partir de la discusión teórica y los antecedentes presentados, se entiende que los factores que contribuyen a los procesos y resultados de la política implementada, en este caso el OLPC Perú, son múltiples y complejos, y no se puede asignar a un solo componente o elemento. A tales efectos, se descompusieron y analizaron las diversas dimensiones que emergen para conocer mejor y poder realizar una caracterización del proceso de desarrollo de esta iniciativa. Por un lado, las formas de implementar la política y la concepción que la fundamenta; en segundo lugar, como se adaptó la propuesta OLPC al contexto; en tercer lugar, el análisis de las características específicas del modelo 1 a 1 , como propuesta diferente a las que se había realizado hasta el momento y sus resultados sobre las desigualdades; y, por último, cuáles fueron los motivos de su interrupción y las consecuencias sobre el problema que se buscaba resolver.

Para los fines propuestos se realizó un análisis del caso peruano en base a un estudio "Revisión comparativa de iniciativas nacionales de aprendizaje móvil en América Latina" ${ }^{1}$, realizado en 2015. El estudio de caso de el Perú, estuvo a cargo de la autora del presente artículo y se sustentó en el análisis de documentos políticos, entrevistas a informantes calificados y de análisis de datos estadísticos sobre la evolución del proceso.

Los documentos políticos analizados son los que produjo el propio Ministerio gubernamental, que implementó esta política; se consultaron leyes presupuestales e informes internos, a los que se pudo acceder; se analizaron datos estadísticos nacionales -del Instituto de Estadística de Perú, de Encuesta de Educación y Tecnologías-, así como del Ministerio. En 2015, se realizaron entrevistas a 12 actores responsables de políticas públicas a nivel de direcciones ministeriales, ex directores actuantes durante el período de implementación y a otros mandos medios. Estas entrevistas tuvieron una duración mínima de una hora; e, incluso, en tres de ellas se realizó más de un encuentro. Se analizaron fuentes privilegiadas de información sobre el proceso para comprender las distintas dificultades y contradicciones. A pesar de proceder todas de fuentes oficiales, al tratarse de gobiernos distintos, se pudieron obtener visiones críticas de las distintas prácticas y resultados.

1 Se trata de una investigación con cinco estudios de caso en América Latina organizado en forma conjunta entre la División de Políticas y Sistemas de Educación Permanente del Sector de Educación de la UNESCO y el Instituto Internacional de Planeamiento de la Educación de UNESCO en Buenos Aires, bajo la coordinación general de María Teresa Lugo y Violeta Ruiz. 


\section{El contexto histórico de la iniciativa OLPC Perú}

El Perú cuenta con una población de 32 millones de personas, el $73 \%$ habita en zonas urbanas y el $27 \%$ en zonas rurales (INEI, 2015). Tiene un $25 \%$ de población de pueblos originarios (Ribbota 2010 ) y cerca del $15 \%$ tiene otra lengua materna -quechua, aimara u otra-. Entre 2000 y 2010 su economía creció a ritmo acelerado en torno al 5\% anual, con estabilidad macroeconómica, mejorando sus indicadores sociales y reduciendo la pobreza extrema del 26 al 6\% en 2012 (INEI, 2015). Tanto la pobreza como la desigualdad persisten como problemas sociales centrales del país, afectando en mayor grado a la infancia, la población indígena, la población rural y a las mujeres.

De acuerdo a lo planteado por Marín et al. (2014), el sector de las TIC y de telecomunicaciones del Perú está experimentando un acentuado proceso de cambio que trae aparejado un nuevo ecosistema digital, lo que ha supuesto un crecimiento exponencial de la telefonía móvil, por el impulso del gobierno de universalizar el acceso a los servicios de banda ancha, el despliegue de la infraestructura de fibra óptica, una nueva gestión del espectro radioeléctrico y la ampliación del acceso a internet.

Al igual que otros países de la región, el Perú experimentó una explosión de las tecnologías digitales. En el año 2013 el 98,1\% del país disponía de cobertura de telefonía móvil, un 5,2\% de banda ancha fija y un 2,9\% de banda ancha móvil. El 32\% de los hogares contaba con computadora y el $22 \%$ con acceso a internet, siendo el 39\% de la población usuarios de internet ${ }^{2}$. Según Marín (2014) estos avances de telefonía móvil contribuyeron a mejorar la falta de acceso y la conectividad, en un país con limitaciones geográficas para la expansión de redes terrestres, sobre todo en áreas rurales.

Las iniciativas y políticas previas a OLPC contribuyeron tanto a la infraestructura como a los contenidos, a la formación a pequeña escala y sobre todo a nivel urbano. Las limitaciones estaban dadas por las condiciones generales de infraestructura del país y por los altos costos de conectividad que esto conlleva. Estas empezaron a funcionar en los años 90, algunas se mantuvieron, pero en la mayoría de los casos fueron discontinuas; lo que según Balarin (2013) se debe a que cada administración toma su propia orientación e iniciativa y, por tanto, cada una se identifica con el gobierno

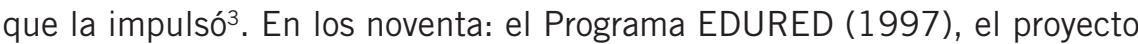

${ }^{2}$ Ver: https://bit.ly/2EdCc65

${ }^{3}$ Las iniciativas existentes se desarrollan durante cuatro administraciones presidenciales: Fujimori en los años noventa, de Alejandro Toledo (2001 y 2006), Alan García (2006 al 2011) y Ollanta Humala (2012-2016). 
INFOESCUELA (1995-2001) y una iniciativa piloto de Educación a Distancia (1998). El Plan Huascarán en 2001 fue uno de los de mayor trascendencia y reconocimiento por la inclusión en su contenido de las culturas y lenguas indígenas y la creación de las Aulas de Innovación Tecnológica (AIT), salas equipadas con computadoras para un doble propósito. El Plan Huascarán es la política pública señalada por los entrevistados de más impacto y de relativo éxito.

\section{One Laptop Per Child - Peru (OLPC)}

El proyecto One Laptop Per Child (OLPC) del Massachusetts Institute of Technology ${ }^{4}$ (MIT) tuvo como objetivo la creación de una laptop para uso educativo. Su costo sería de 100 dólares y el prototipo se logró en el año 2006. Esta propuesta se sustentó en la idea de Nicholas Negroponte de producir computadoras a bajo costo, accesibles para los gobiernos, de forma que pudieran acceder a las TIC niños y niñas pobres. En definitiva, bajo el supuesto de que favorecería la reducción de la brecha digital y las desigualdades sociales a ella vinculadas (desarrollo del aprendizaje, autonomía, mejores condiciones de trabajo y para la educación). Bajo este fundamento constructivista, Seymour Papert apostaba fuertemente en la autonomía de los niños para su formación mediante el uso de la tecnología con independencia de los docentes. La propuesta fue presentada en la Cumbre de Sociedad de la Información organizada por Naciones Unidas en 2005 y comenzaron a implementarse experiencias piloto en distintos países.

Esta iniciativa comenzó en 2007 bajo la presidencia de Alan García (2006-2011), coordinada por el Ministerio de Educación (MINEDU), implicó la distribución universal de laptops bajo la modalidad de una computadora por niño de OLPC a partir de la educación pública, lo que constituyó una de las mayores inversiones públicas del país en este sector ${ }^{5}$. En el documento del proyecto se fundamenta que:

“... si los niños de nuestras áreas rurales no reciben hoy urgentemente una educación moderna y de calidad, cuando sean adultos no podrían entender ni aprovechar el mundo que les toque vivir, quedando condenados a ser -en el mejor de los casos - sólo mano de obra barata para servicios menores." (DIGETE, 2008).

${ }^{4}$ Ver: http://web.mit.edu/

${ }^{5}$ Ver: https://bit.ly/1EmFzjV 
Según manifiesta el director de la iniciativa, para su creación se fundamentó en la importancia de las TIC en la educación y en la ausencia de una política universal para su acceso, con el fin de subsanar el bajo acceso a internet en los hogares y en la calidad docente. Cabe destacar que surgió de forma independiente, pues no estaba prevista en la Agenda Digital de Perú de este período y por tanto no estaba previsto en su presupuesto (CODESI, 2005).

Para lograr el acuerdo se negociaron algunos requisitos de OLPC que no se podían cumplir. Por ejemplo, la disminución de la cantidad de dispositivos con que se iniciaría la experiencia por razones presupuestarias ${ }^{6}$. Se dio prioridad a las poblaciones rurales en escuelas unidocentes multigrado. Por otro lado, sólo el $2 \%$ de las escuelas contaban con acceso a internet por lo que se debió acordar su implementación sin internet.

Según uno de los entrevistados, también se argumentó que la calidad docente no era la adecuada y la iniciativa permitiría que los estudiantes pudieran aprender con independencia de la formación docente, facilitados por el uso del dispositivo. Sin embargo, en los objetivos formulados como políticas del documento (DIGETE, 2008), se hace referencia en generar capacidad de gestión pedagógica, desarrollar capacidades, habilidades y destrezas de los estudiantes y de capacitación a los docentes

Se registran acciones del OLPC de 2007 a 2012. A partir del análisis de la documentación y de las entrevistas, hubo cambios significativos en la política, debido a decisiones que conformaron hitos durante el proceso de implementación, pueden separarse en cuatro fases.

1. Etapa de la prueba de concepto, iniciada a partir de la donación de 200 computadoras por parte del MIT y su ejecución en una escuela de Araguay. Según el director, buscando que las condiciones fueran lo menos artificiales posibles, para asegurar la puesta en marcha del proyecto. Se realizó en una área rural con una escuela multigrado de pocos alumnos. Se le dio conexión a internet, se hizo una breve demostración de cómo usar las XO a los docentes y se entregaron las computadoras. Se buscaba verificar la viabilidad y el beneficio, para evaluar la implementación como política. Después de una evaluación, a los pocos días, se constató el uso por parte de los niños y se decidió la compra de las computadoras y extender la experiencia.

${ }^{6}$ El precio inicial de las computadoras era de 100 dólares, luego modificado 
2. Etapa del lanzamiento de "One Laptop Per Child" Perú. Se definió el alcance del proyecto, que consistió en la entrega una laptop XO a cada estudiante de instituciones educativas unidocentes del nivel primaria, ubicadas en áreas rurales que sumaban 200.000 alumnos distribuidos en 22.000 centros. En 2008 se entregaron $40.000 \mathrm{XO}^{7}$ a los estudiantes de 500 escuelas rurales que tenían electricidad e internet, en "Cesión en uso" por lo que no se podía comercializar y en caso de pérdida o rotura no se repondría.

Esta etapa, a efectos de entrega, fue de un gran esfuerzo logístico por parte del MINEDU, estuvo facilitado por la autonomía financiera. A partir de lo manifestado por otros entrevistados, se vincularon las entregas a los tiempos políticos, buscando la presencia del ministro o gobernador de turno. Estas instancias, de gran popularidad, daban una amplia difusión mediática y, por tanto, gozaban de un gran valor simbólico y político.

Los entrevistados manifestaron que la infraestructura era bastante deficitaria, ya que algunas escuelas ni siquiera contaban con electricidad. La falta de conectividad era conocida de antemano y se intentó sustituir a partir de una conectividad asincrónica, mediante un dispositivo de memoria USB que los maestros debían actualizar. Con posteridad se constataría que esta solución no funcionó como se esperaba.

Según analizan Olivera y Villanueva (2013), la entrega de las computadoras fue de gran emoción y expectativa entre la población, sobre todo por los niños, fue como un aterrizaje a la modernidad. Pero, también de mucho temor, por parte de los padres y los responsables de los centros educativos, por la responsabilidad que implicaba la rotura o pérdida, por lo que en muchos casos se restringía su uso. Según el Área de Monitoreo de la DIGETE, en el año 2008, su encargado constató que entre el 25 y el $30 \%$ de las computadoras ni siquiera habían sido desembaladas.

3. Etapa de la universalización de la iniciativa. Comienza en noviembre de 2009, ampliándose a los centros polidocentes y multigrado de nivel primario. Se crean los Centros de Recursos Tecnológicos (CRT) para estimular el uso de recursos y herramientas de apoyo al desarrollo curricular, en aquellos centros que contaban con conexión a internet, se incorporaron otros

\footnotetext{
${ }^{7}$ Ver: https://bit.ly/2XpGorQ
} 
elementos informáticos, como módems y puntos de acceso para la conexión inalámbrica. Se tomó esta decisión debido a los elevados costos que suponía mantener el modelo 1 a 1.

"EI CRT es un sitio donde hay tecnología no es un sitio físico, no es una habitación es un concepto. De manera que los niños pueden tener la máquina, para usar la como quieran, al menos cuatro horas a la semana, dos horas en periodo de clase y dos horas fuera de clase. [...] Vamos a ponerles kit de robótica para que los chicos trabajen en grupo y entonces un maquina sirve para 4 o 5 chicos porque están haciendo distintas tareas cada uno y una impresora así."

Según la Ley de Presupuesto 29.109, del 29 de octubre de $2007^{8}$ se establece un crédito suplementario para adquirir laptops y se le otorga una amplia autonomía al MINEDU para su manejo. Se crea la Dirección General de Tecnologías Educativas (DIGETE) para la ejecución del Programa con el fin de coordinar los distintos organismos, brindar conectividad, implementar plataformas tecnológicas y educación a distancia, entre otros ${ }^{9}$. Esto facilitó su ejecución, pero fue muy criticada por no cumplir con los procedimientos regulares en las adquisiciones públicas, dado que no eran ingresados al Sistema Nacional de Inversión Pública (SNIP). Según señalan Olivera y Villanueva (2013) se hace referencia a una Fundación OLPC cuyo cometido era recibir contribuciones privadas para la iniciativa, pero no se encontró referencia a ella en la documentación.

La información sobre la logística de la distribución de los equipos es escasa, pero se evidencia que fue dificultosa y no libre de problemas (Laura, 2011). La complejidad de la gestión y el aumento de las responsabilidades de la DIGETE se facilitaban por la centralidad en la toma de decisiones, pero con grandes dificultades para la coordinación interinstitucional, con problemas emergentes de mantenimiento, formación docente, de personal y generación de contenidos, entre otros. Derndorfer (2010) concluye que esta debilidad institucional inicial y las limitaciones para generar mejores condiciones para su sostenibilidad, fue uno de los principales problemas.

4. Etapa de la ampliación a educación secundaria y finalización. En 2010 se amplía a la educación secundaria en el marco de los CTR y se adquieren las computadoras Magallanes ${ }^{10}$, que tienen

8 Ver: https://bit.ly/2TgTOCG

${ }^{9}$ Decreto Supremo No 016-2007-ED, ver: https://bit.ly/2T12Pp1

10 Ver: https://bit.ly/2TcOqW1 
más memoria y son más rápidas. Se recibieron antes del cambio de gobierno y fueron distribuidas por el gobierno que entró en 2012. En este periodo se produjo un incendio en el depósito, perdiéndose miles de dispositivos adquiridos (máquinas, servidores, proyectores, sensores, etc.) ${ }^{11}$. A las circunstancias del incendio, se sumó la publicación de un informe de seguimiento y monitoreo, elaborado por el Banco Interamericano de Desarrollo a solicitud de la DIGETE. Haciéndose públicos los resultados insuficientes de la iniciativa y recibiendo críticas importantes. El director de la DIGETE del nuevo gobierno informó que no se iban a realizar más compras de equipos y que no se continuaría con el proyecto, tal como se estaba desarrollando, sino que se buscaría sostenibilidad, uso pedagógico y conectividad en trabajo conjunto con otros actores ${ }^{12}$. No obstante, sobre 2016 el presidente Ollanta Humala vuelve a realizar una entrega masiva de laptops.

A partir de estas fases se evidencia que OLPC Perú, a lo largo de su existencia, va mutando en algo diferente, por tener que adaptarse a la realidad peruana, sus posibilidades presupuestarias y de conectividad. De manera progresiva, se abandona la modalidad 1 a 1 en el acceso a tecnología móvil que se transforma en el acceso sólo disponible en los centros educativos.

El monitoreo y seguimiento de la iniciativa constituyó un factor crítico para su evaluación, pues no se realizaron informes ni existen muchos datos al respecto. Los entrevistados sostienen que esto fue una debilidad. Los esfuerzos estuvieron concentrados en la distribución y el financiamiento, orientado a la compra de equipos.

Una aproximación a la inversión realizada puede obtenerse a partir de distintas fuentes. De hecho, un informe para el Ministerio de Finanzas señala que en el año 2011 se ejecutaron 111.617 .235 soles, siendo la mayor cantidad invertida durante el año 2010 con 282.507.887 soles (Del Mastro Vecchione, 2012). El diagnóstico del "Plan de Desarrollo de la Sociedad de la Información en el Perú La Agenda Digital 2.0" constata que el Proyecto OLPC invirtió 693.165.473 soles, entre los años 2007 y 2011 (CODESI, 2011). Además se señalan los gastos desde otra perspectiva y en costos por estudiante, a la vez que los compara con la inversión por estudiante durante todo el período.

11 La información sobre el total de las pérdidas es imprecisa, la prensa las estima en US\$108 millones. Ver por ejemplo: https://bit.ly/2Sp0Mpl

12 Entrevista a Sandro Marcone. Disponible en: https://bit.ly/2U6vOnT 
Un directivo del MINEDU sostiene con respecto a la revisión de los gastos realizados por OLPC:

"No había un texto programático, no se ingresó la idea o voluntad al formato de Proyecto de Inversión Pública siquiera. Pese a eso los montos de inversión no fueron menores... el cálculo que hicimos fue que el verdadero costo al gobierno de cada unidad fue de casi 250 dólares (el precio oficial del contrato decía 188 dólares). Durante esos años (2007-2011) la inversión por alumno sin contar equipamiento no llego ni a los 80 dólares en total, ni siquiera por año."

Se adquirieron cerca de 900.000 laptops, más varios miles de kit de robótica, access points, usb, etc. Se cubrió el 90\% de las escuelas primarias y el $21 \%$ de secundaria (ODETIC, 2015). La Encuesta Nacional de Educación (ENEDU, 2011) indica que sólo el 0,1\% de primer y segundo grado de las escuelas unidocentes de primaria contaban a 2011 con internet. En 2011, el $90,4 \%$ de la computadoras de primaria estaban en funcionamiento. En la tabla 1 se muestra los tipos de dispositivos distribuidos.

Tabla 1 Cifras de la infraestructura y equipos entregados del programa Una Laptop por Niño Perú.

\begin{tabular}{lr}
\hline Tipo de dispositivo y destino de su distribución & Cantidad \\
\hline Laptop XO - Nivel Primaria & 585.867 \\
Laptop XO - Nivel Secundaria & 227.029 \\
Classmate - Nivel Primaria & 1.672 \\
Classmate - Nivel Secundaria & 27.809 \\
Kits de Robótica - Nivel Primaria & 83.704 \\
Servidores - Nivel Primaria & 5.654 \\
Estación de trabajo - Primaria y Secundaria & 775 \\
Access Point - Nivel Primaria & 5.654 \\
Access Point - Nivel Secundaria & 5.654 \\
USB - Nivel Primaria & 81.293 \\
Proyectores/ ECRAN - Nivel Primaria & 5.624 \\
Notebook - Nivel Primaria & 5.624 \\
Laptop para Reposición & 31.416 \\
\hline Fuente: Oficina de Tecnologías de la Información y la Comunicación del Ministerio de \\
Educación (2015).
\end{tabular}

Los distintos informes resaltaron otros elementos, cómo que solo el $56,9 \%$ de los niños se llevaba a casa la computadora, que su uso por parte de los niños era inmediato y que en más del $90 \%$ se producían algunos elementos favorables para su desarrollo cognitivo y facilitador del aprendizaje, aunque no se evidenciaron mejoras en la Matemática y la Lengua; desarrollaron habilidades tecnológicas y se hicieron más reflexivos sobre su aprendizaje y desempeño, y que hubo mejores expectativas de los maestros y los padres sobre la educación de los niños (Santiago et al., 2010; Cristiá et al., 2012). 
En 2013 el 99\% de las escuelas primarias y el 86\% de secundaria tenían, al menos, un equipo de la laptop XO, aumentando del $10 \%$ al $36 \%$, y un $29 \%$ en secundaria las que estaban fuera de funcionamiento. El $25 \%$ de los centros contaba con conexión y sólo el 9\% en la clase (ENTIC, 2011 y 2013). Las dificultades y costos para que las empresas dieran conexión fue uno de los factores clave para la instauración del sistema digital.

Tabla 2. Población que accede a internet ${ }^{13}$

Población de 6 y más años de edad que hace uso de internet, según lugar de uso, grupos de edad y ámbito geográfico, 2009-2013

\begin{tabular}{|c|c|c|c|c|c|}
\hline & 2009 & 2010 & 2011 & 2012 & 2013 \\
\hline \multicolumn{6}{|l|}{ Cabina Pública } \\
\hline 6-16 años & 67.8 & 64.0 & 58.1 & 50.3 & 46.7 \\
\hline 17-24 años & 65.3 & 58.7 & 50.7 & 46.8 & 41.1 \\
\hline 25 y más años & 40.6 & 37.9 & 30.7 & 25.8 & 22.4 \\
\hline \multicolumn{6}{|l|}{ Hogar } \\
\hline 6-16 años & 14.5 & 17.8 & 22.7 & 27.9 & 30.8 \\
\hline 17-24 años & 13.3 & 15.6 & 19.7 & 22.4 & 22.3 \\
\hline 25 y más años & 19.9 & 22.8 & 27.2 & 30.6 & 32.2 \\
\hline \multicolumn{6}{|l|}{ Trabajo } \\
\hline 6-16 años & 0.1 & 0.1 & 0.1 & 0.1 & 0.1 \\
\hline 17-24 años & 2.3 & 2.7 & 2.7 & 2.3 & 2.2 \\
\hline 25 y más años & 12.7 & 11.2 & 11.0 & 10.5 & 9.2 \\
\hline \multicolumn{6}{|l|}{ Hogar y Trabajo } \\
\hline 6-16 años & 0.1 & 0.1 & 0.0 & 0.0 & 0.0 \\
\hline 17-24 años & 1.6 & 1.5 & 2.3 & 2.5 & 1.8 \\
\hline 25 y más años & 11.3 & 11.2 & 13.9 & 12.5 & 10.4 \\
\hline \multicolumn{6}{|l|}{ Colegio y Cabina Pública } \\
\hline 6-16 años & 5.4 & 3.8 & 2.7 & 3.6 & 2.7 \\
\hline 17-24 años & 3.9 & 4.3 & 3.5 & 2.7 & 2.1 \\
\hline 25 y más años & 0.9 & 0.8 & 0.6 & 0.5 & 0.3 \\
\hline \multicolumn{6}{|l|}{ Trabajo y Cabina Pública } \\
\hline 6-16 años & 0.0 & 0.1 & 0.1 & 0.0 & 0.0 \\
\hline $17-24$ años & 1.8 & 1.3 & 1.5 & 1.1 & 0.6 \\
\hline 25 y más años & 5.6 & 4.9 & 3.6 & 2.7 & 2.1 \\
\hline \multicolumn{6}{|l|}{ Hogar y Cabina Pública } \\
\hline 6-16 años & 1.9 & 1.5 & 2.0 & 1.8 & 1.7 \\
\hline $17-24$ años & 2.9 & 2.8 & 3.2 & 2.6 & 2.2 \\
\hline 25 y más años & 2.2 & 2.2 & 1.8 & 1.8 & 1.5 \\
\hline \multicolumn{6}{|l|}{ Colegio } \\
\hline 6-16 años & 2.7 & 2.6 & 3.2 & 3.2 & 3.3 \\
\hline $17-24$ años & 1.4 & 1.3 & 1.2 & 1.5 & 1.5 \\
\hline 25 y más años & 0.5 & 0.3 & 0.3 & 0.2 & 0.3 \\
\hline \multicolumn{6}{|l|}{ Hogar y Colegio } \\
\hline 6-16 años & 2.4 & 3.1 & 3.8 & 4.3 & 3.6 \\
\hline 17-24 años & 1.8 & 2.5 & 3.1 & 3.1 & 2.2 \\
\hline 25 y más años & 0.3 & 0.8 & 0.5 & 0.4 & 0.4 \\
\hline \multicolumn{6}{|l|}{ Otro lugar } \\
\hline 6-16 años & 5.1 & 7.1 & 7.2 & 8.7 & 11.0 \\
\hline $17-24$ años & 5.8 & 9.2 & 12.1 & 15.1 & 24.0 \\
\hline 25 y más años & 6.0 & 7.9 & 10.3 & 15.1 & 21.3 \\
\hline
\end{tabular}

Fuente: INEI (2015)

13 Ver: https://bit.ly/2GKqZO6 
De acuerdo a lo que se muestra la tabla 2, el impacto del OLPC en las cifras de acceso en los centros educativos es muy insignificante. En el período durante el cual se implementó la política, el aumento en el tramo etario de edad escolar fue más significativo en "otros", donde se hace evidente por el aumento de la difusión de la telefonía celular en el período.

En suma, como resultado se observa que la iniciativa proveyó de equipamiento tecnológico a gran parte de los centros. A su vez contó con una visión positiva de los actores sobre el beneficio del uso de las TIC para el aprendizaje. También la importancia de la voluntad política para que la tecnología fuera incorporada a la agenda educativa. Finalmente, la importancia de brindar no sólo los dispositivos sino también la conectividad y el acceso a recursos, además de la formación necesaria para su aprovechamiento. Cabe destacar, desde el punto de vista de la implementación de políticas, la importancia de la continuidad de las mismas, según fueron transcendiendo períodos de gobierno; así como su debida evaluación, seguimiento y monitoreo, que permitió valorar en su debido tiempo la adecuación de las inversiones realizadas para hacer los ajustes necesarios.

\section{Discusión}

La implementación del proyecto One Laptop Per Child, entre 2008 y 2012, conformó una novedad por lo ambicioso de los objetivos, fundamentalmente por el pretendido alcance universal de dicha iniciativa. Como parte de sus fundamentos se propuso contribuir en la disminución de las desigualdades existentes como eje de acción en términos de brecha digital y su negativo impacto en el futuro de los estudiantes. La pertinencia de este objetivo es evidente a partir de los bajos índices de acceso a internet del país en la época y en particular en los centros educativos.

A partir del análisis del caso, se constatan algunos elementos característicos en la ejecución política. En primer lugar, la iniciativa partió del más alto nivel de decisión: la Presidencia del país. Luego encomendada al Ministerio de Educación donde se centralizó su aplicación en la Dirección General de Tecnologías Educativas, creada especialmente con tales fines. Este aspecto tuvo el valor de contar con un gran peso político en cuanto a decisión por su centralidad en la ejecución, pero también emergió el problema de la falta de coordinación con otros ámbitos, no enmarcados en la estrategia de desarrollo explicitada en la materia, por lo que supuso una tarea titánica para una sola unidad administrativa. A su vez, surgieron cuestionamientos por la falta de control en la administración, de evaluación y de seguimiento. La falta de un diagnóstico previo y una planificación fueron factores que agudizaron 
esta situación. Además hubo problemas de actualización, mantenimiento y reciclado de desechos electrónicos, que una política de este alcance debe prever. Aunque, con retrasos y dificultades las computadoras llegaron a destino.

Desde la fundamentación, en su ambición de universalidad y reducción de la brecha digital a nivel de la población, pues no sólo fue pensado para el sistema educativo, el programa fue cambiando. A partir del análisis de sus distintas etapas, se constató que los ajustes se debieron a condiciones estructurales; como el déficit en infraestructura, en especial, en el acceso a internet, en otros casos también a la energía eléctrica. Si bien, algunos fueron subsanados, no en todos los casos fue posible por los altos costos implicados. Esto obligó a cambiar una de las principales características del proyecto, que era el acceso a internet de las computadoras. El perfil de la propuesta fue cambiando de una modalidad de 1 a 1 en forma universal, a la de priorizar el sector más excluido, que eran las escuelas rurales. Esta decisión se vinculó a la voluntad de contemplar a los más excluidos, pero también implicó afrontar las dificultades mencionadas por las características del contexto precario en términos socioeconómicos. Otro criterio que también cambió fue la distribución a los centros educativos en vez de a cada niño en propiedad.

La compra y distribución de dispositivos fue la actividad central del proyecto. La formación docente, los contenidos digitales adecuados, el desarrollo de la conectividad y el uso efectivo de los dispositivos no estuvieron presentes en las acciones ejecutadas. En parte, debido al desborde logístico, pero también puede adjudicarse a la concepción inicial que apuntaba, que el mero acceso a la tecnología por parte de los niños por sí solos, provocaría un mejor desempeño y aprendizaje, independientemente del docente. Quizá influido por la creencia que el solo hecho de acceso produce cambios.

Esto sin desmedro que un país con las condiciones sociales y étnicas del Perú, debiera considerar sin dudas políticas incluyentes en este sentido a los efectos de no reforzar desigualdades preexistentes y fomentar el uso de las tecnologías de forma contextualizada. La dimensión étnica había estado presente en políticas de TIC anteriores en el país pero no en esta experiencia. Esto evidencia una ruptura en la continuidad de aprendizaje institucional que es también característico de estas políticas.

El programa estuvo centrado en el equipamiento, pero la débil infraestructura a nivel nacional, así como la ausencia de electricidad en las zonas priorizadas implicó la redefinición del proyecto. Esto condicionó fuertemente el aprovechamiento del recurso debido a la importancia que tiene internet para el desarrollo de nuevas prácticas como incentivo para el uso y la apropiación, que hace atractivo el modelo de aprendizaje móvil, no solo a los estudiantes sino también a sus familias. 
Algunos elementos encontrados van claramente en el sentido opuesto de la reducción de las desigualdades. El problema de la infraestructura y conectividad a nivel nacional y la imposibilidad de solucionar este aspecto antes de la implementación de un programa y modelo pedagógico que lo presupone, es uno de los elementos a ser considerado seriamente en estos programas. Se desaprovecha el recurso, no estimula su uso en el hogar ni en el aula tanto a estudiantes como a docentes. Por tanto, las condiciones de infraestructura y acceso junto con el dispositivo, siguen constituyendo un peso en la reducción de la brecha que tiene temas pendientes en sociedades como las latinoamericanas.

La expansión de la telefonía móvil y en particular la irrupción de los teléfonos inteligentes interpelan en parte estas políticas. Pero esto también -o, al menos- amerita la reflexión, porque los dispositivos se elaboran con distintos fines y sus posibilidades difieren. Por una parte, no hay consenso en los actores educativos respecto del uso de celulares en el aula y, a su vez, no todos tienen celulares de calidad en los contextos latinoamericanos.

Este estudio se suma a los hallazgos de otros estudios respecto de las limitaciones del modelo pedagógico de OLPC en lo relativo a la apuesta a que los niños consoliden el proceso de aprendizaje de forma independiente al docente. Si bien el rol docente debe experimentar en la actualidad una redefinición a la luz de las tecnologías digitales móviles, de este estudio se desprende que este recurso digital se desaprovecha mucho si no se cuenta con el compromiso de este actor educativo. Los estudiantes perdieron el entusiasmo inicial y no se sostuvo el uso en el tiempo. Esto se debió también a los temores por rotura de las instituciones y los hogares dada la falta de conocimiento, ya que estaba previsto el mecanismo de reparación. La centralidad del docente y preparación básica para su incorporación en el aula, así como la de orientar su uso es clave.

En conclusión, la política implementada buscó a partir de su originalidad e innovación disminuir el grave problema de acceso y conectividad por parte de la población más excluida y reducir las desigualdades de acceso a las tecnologías, así como mejorar la educación. Se encontraron usos y testimonios del beneficio por el contacto con las tecnologías digitales, pero poco significativos, pues no se evidenció reducción de la brecha digital. Corresponde en tal sentido, cuestionar los resultados de la política en relación al enorme esfuerzo de gestión para su ejecución como en recursos invertidos. La inadecuación de la iniciativa en relación al contexto de infraestructura limitada del país, así como la mutación del modelo 1 a 1 a lo largo de la implementación, evidenciaron la importancia de la adecuación de las iniciativas al contexto de infraestructura, institucional y cultural para que los resultados constituyan aportes reales en la reducción de desigualdades. 
En el estudio comparado de distintas experiencias de políticas públicas similares, en futuras investigaciones, sería conveniente comprender mejor los factores diferenciales, que hacen posible fracasos y éxitos en términos de propósitos de reducción de desigualdades.

\section{Referencias bibliográficas}

Albertos, A., A. Domingo y J. E. Albertos (2016). Estrategia docente para el desarrollo de la competencia digital en el aula universitaria: del uso recreativo al uso formativo. Educar 52(2).

Balarin, M. (2013). Las Políticas TIC y los sistemas educativos de América Latina: Caso Perú. Unicef. https://uni.cf/1RBAilm

Benitez, S. y R. Winocur (Coord.) (2016). Inclusión Digital. Una mirada crítica sobre la evaluación del Modelo uno a uno en Latinoamérica. Editorial Teseo, Argentina.

Bonilla, M. y G. Cliche (2001). Internet y sociedad en América Latina. Fundación Acceso. Costa Rica.

Cabero, J. y Llorente, M.C. (2013). La aplicación del juicio de experto como técnica de evaluación de las tecnologías de la información (TIC). Revista de Tecnología de Información y Comunicación en Educación, 7 (2), 11-22. Recuperado de https://bit.ly/2EzAtJL

Camacho, K. (2001). Internet: ¿una herramienta para el cambio social? Elementos para una discusión necesaria. https://bit.ly/2Sr3ZFd

Castells, C. (2002). The Internet galaxy: Reflections of the Internet, business and society. Oxford: Oxford University Press.

Cano, A. M. (2015). Digital technology in public education: One Laptop Per Child programme in Perú. En: Ed. Sara Pereira (2015) Digital literacy, Technology and social inclusion. Making sense of one-to-one computer programmes around the world. Portugal : Humus .

Claro, M. et al. (2011). Aporte del sistema educativo a la reducción de las brechas digitales. Una mirada desde las mediciones PISA. Documentos de Proyectos Nº 456 (LC/W.456), Santiago de Chile, CEPAL.

CODESI (2005). Plan de Desarrollo de la Sociedad de la Información en el Perú. La Agenda Digital Peruana. Comisión Multisectorial para el Desarrollo de las Sociedad de la Información), Lima.

CODESI (2011). Plan de Desarrollo de la Sociedad de la Información en el Perú La Agenda Digital 2.0. Comisión Multisectorial para el Seguimiento y Evaluación del Plan de Desarrollo de la Sociedad de la Información Disponible en https://bit.ly/1zf180N

Cortés, J. y A. Dubois (2005). Nuevas Tecnologías de la Comunicación para el desarrollo humano. https://bit.ly/1nW2gcd

Cristiá, J.; Ibarrarán, P.; Cutelo, S.; Santiago A. y Severín, E. (2012). Technology and child development. Evidence of the One Laptop Per Child program. Banco Interamericano de Desarrollo.

Del Mastro Vecchione, C. (2012). Informe final evaluación de diseño y ejecución de presupuesto de: Una Laptop por niño. Unidad de coordinación de Préstamos Sectoriales - UCPS Ministerio de Economía y Finanzas - MEF 
Derndorfer, C. (2010). OLPC in Perú: A Problematic Una Laptop Por Niño Program. https:// bit.ly/R9TOzR

Duart, J., Gil. M., Pujol, M. y Castaño, J. (2008). La Universidad en la sociedad RED, usos de la internet en educación superior, Barcelona: Ariel.

DIGETE (2008). Programa "una laptop por niño" se fortalece, estudiantes y docentes recibirán acompañamiento pedagógico. https://bit.ly/2GMjZQJ.

ENEDU (2011). Encuesta Nacional de Educación. Instituto Nacional de Estadísticas de Perú.

ENTIC (2013). Encuesta Nacional de Tecnologías de la Información. Ministerio de Educación.

García, I.; Gros, B. y Escofet, A. (2012). La influencia del género en la cultura digital del estudiantado universitario. Athenea Digital, 12(3).

Gascó-Hernández, M., F. Equiza-López y M. Acevedo-Ruiz (2007). Information Communication Technologies and Human Development: Opportunities and Challenges. Idea Group Publishing.

Griffin, P., B. McGaw y E. Care (2012). Assessment and teaching of 21st century skills. Springer.

Hargittai, E. y A. Hinnant (2008). Digital inequality: Differences in young adults' use of the Internet. Communication Research, 35(5).

Hargittai, E. (2004). Internet access and use in context. New Media and Society, 6 (1): 115-21.

Helsper, H. (2012). A corresponding fields model for the links between social and digital exclusion. Communication Theory, 22 (4). pp. 403-426.

Hinostroza, J. E. y Labbé, C. (2011). Políticas y prácticas de informática educativa en América Latina y El Caribe. División de Desarrollo Social de la Comisión Económica para América Latina y el Caribe (CEPAL). Santiago. Naciones Unidas (Serie Políticas Sociales).

INEI (2015). Instituto Nacional de Estadística e Informática de Perú. Boletín Estadístico: Indicadores económicos y sociales- año 5/ edición 03- Marzo 2015. Disponible en: http://www.inei.gob.pe/estadisticas/indice-tematico/education/

Jara, I. (2011). Identificación de buenas prácticas de Proyectos de TIC para la Educación. Documento de trabajo. Proyecto @LIS 2 División de Desarrollo Social, CEPAL.

Laura, C. D. (2011). Una laptop por niño en escuelas rurales del Perú: Un análisis de las barreras y facilitadores. Sexto Encuentro Iberoamericano de colectivos escolares y redes de maestros/as que hacen investigación e innovación desde su escuela y comunidad - Argentina. Disponible en: https://bit.Iy/2VjoxB9

Mansell, R. (2002). From Digital Divides to Digital Entitlements in Knowledge Societies. Current Sociology, 50(3), 407-426.

Marín, J., Barragán, X. y Zaballos, A. (2014). Informe sobre la situación de conectividad de Internet y banda ancha en Perú. Banco Interamericano de Desarrollo.

MINEDU (2013). PISA 2012: Primeros resultados. Informe Nacional del Perú. https://bit.ly/1tORuM9

Mística (2003). Comunidad Virtual Trabajando la Internet con visión social. En: Otro lado de la Brecha: Perspectivas latinoamericanas y del Caribe ante la CMSI. RedISTIC, Caracas.

Norris, P. (2001). Digital Divide. Civic engagement, information poverty and Internet worldwide. Cambridge University Press.

ODETIC (2015). Informe de la Oficina de Tecnologías de la Información y la Comunicación del Ministerio de Educación (2015). 
Olivera, P. y E. Villanueva (2013) Barreras Institucionales para el Desarrollo de una Innovación: Evaluando la Implementación de las Computadoras XO en dos Escuelas Periurbanas del Perú. https://bit.ly/2Ec4Vbj

Pedró, F. (2006). The new millennium learners: Challenging our Views on ICT and Learning. https://bit.ly/1NAVU7B

Rivoir, A. (2013). Enfoques dominantes en las estrategias para la sociedad de la información y el conocimiento: el caso uruguayo 2000-2010. Revista de Ciencias Sociales, 33, 11-30.

Rivoir, A. (2015). La desigualdad digital a la luz de las iniciativas para su reducción. https:// bit.ly/2H83EF4

Rivoir, A. (2017). ¿Desarrollo informacional a la uruguaya? Constataciones y contradicciones del período 2015 - 2014. En: A. Rivoir (Coord.) Tecnologías Digitales en Sociedad. Análisis empíricos y reflexiones teóricas. Montevideo: CSIC Biblioteca Plural.

Rivoir, A. y S. Lamschein (2018). Desafíos para el estudio de las desigualdades digitales. Uso, habilidades y resultados tangibles. En: El Uruguay desde la Sociología 15. Departamento de Sociología, Universidad de la República, 81-94.

Robinson, J.P.; DiMaggio, P. y Hargittai, E. (2003). New Social Survey Perspectives on the Digital Divide, IT\&Society, (1)5.

Santiago, A.; Severin, E.; Cristia, J.; Ibarrarán, P.; Thompson, J. y Cueto S. (2010). Evaluación experimental del programa "una laptop por niño" en Perú. Revista Aportes, 5, Banco Interamericano de Desarrollo.

Sassi, S. (2005). Cultural differentiation or social segregation? Four approaches to the digital divide. New Media \& Society, 7(5), 684-700.

Selwyn, N. (2004). Reconsidering Political and popular understanding of the Digital Divide. New Media \& Society, 6(3) 341-362.

Selwyn, N. (2013). Education in a Digital World: Global Perspectives on Technology and Education. London: Routledge.

Stewart, C.; Gil-Egui, G. y Tian, Y. (2006). Framing the digital divide: a comparison of US and EU policy approaches. New Media \& Society 2006, 8(5), 731-751.

Sunkel, G., D. Trucco y A. Espejo (2013). La integración de las tecnologías digitales en las escuelas de América Latina y el Caribe. Una mirada multidimensional. Comisión Económica para América Latina. Santiago de Chile.

UNESCO (2013). Directrices para las políticas de aprendizaje móvil. Organización de las Naciones Unidas para la Educación, la Ciencia y la Cultura. Francia.

Valente, J. A. (2014). Aprendizagem e mobilidade: os dispositivos móveis criam novas formas de aprender? En: Biancocini, M. E.; Medeiros, D. R. y Vilela, S. (2014) Web currículo: Aprendizagem, pesquisa e conhescimento com o uso de tecnologías digitais. Editorial LetraCapital, Serie @aprenersempre.com. Brasil.

Van Deursen, A. y Van Dijk, J. (2014). The digital divide shifts to differences in usage. New Media \& Society, 16(3).

Van Dijk, J. (2005). The Deepening Divide, Inequality in the Information Society. Sage Publications, Thousand Oaks CA, London, New Delhi.

Warschauer, M. (2003). Technology and social Inclusion: Rethinking the Digital Divide. Massachusetts Institute of Technology Press. 